\title{
Characteristics of sagittal spinopelvic alignment in asymptomatic Han Chinese adults
}

\author{
ZHENG ZENG ${ }^{1,2}$, YONG HAI ${ }^{2}$, YUNFENG BI ${ }^{3}$, BING WANG $^{1}$, MIAOMIAO LIU ${ }^{4}$ and YANG LIU ${ }^{1}$ \\ ${ }^{1}$ Department of Orthopedic Surgery, Beijing Tiantan Hospital, Capital Medical University, Beijing 100050; \\ ${ }^{2}$ Department of Orthopedic Surgery, Beijing Chaoyang Hospital, Capital Medical University, Beijing 100020; \\ ${ }^{3}$ Department of Rehabilitation Medicine, The Affiliated Hospital of Qingdao University, Qingdao, Shandong 266000; \\ ${ }^{4}$ Department of Radiology, Beijing Tiantan Hospital, Capital Medical University, Beijing 100050, P.R. China
}

Received January 26, 2018; Accepted August 2, 2018

DOI: $10.3892 / \mathrm{etm} .2018 .6680$

\begin{abstract}
The aim of the present study was to investigate patterns of spine sagittal alignment in asymptotic patients using radiological analysis. A number of studies focused on investigating normal patterns of spinal and pelvic alignment in Chinese populations, while few have evaluated Caucasian and other Asian populations. A total of 10 relevant pelvic and spinal parameters, including pelvic incidence (PI), pelvic tilt (PT), sacral slope (SS), lumbar lordosis (LL), cervical lordosis (CL), thoracic kyphosis (TK), sagittal vertical axis (SVA), lumbopelvic lordosis angle (PR-T12), spino-sacral angle (SSA) and sacral pelvic angle (SPA), were evaluated in asymptomatic Chinese adults from Beijing, aged 21-65 years. Correlations among these parameters, as well as between these parameters and age, and associations with gender and body mass index (BMI) were assessed using Pearson's correlation

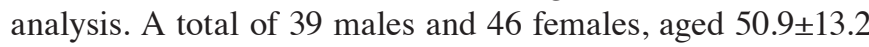
and $41.8 \pm 13.6$ years, respectively, were enrolled in the present study. The results of PI, PT, SS, LL, TK, CL, SVA, PR-T12, SSA and SPA values suggested that age was positively correlated with TK, CL and SVA, while it was negatively correlated with LL, PR-T12 and SSA. SSA and SVA were revealed to differ significantly between sexes. It was also demonstrated that LL and TK may be associated with BMI. In conclusion, the results of the present study indicate that spinopelvic parameters in the asymptomatic Chinese Han population (in Beijing) are correlated with age and may be associated with individuals' ethnicity, sex and BMI. The results of the current study may provide a basis for the restoration of LL or similar diseases.
\end{abstract}

Correspondence to: Dr Yong Hai, Department of Orthopedic Surgery, Beijing Chaoyang Hospital, Capital Medical University, 8 Gongti South Road, Chaoyang, Beijing 100020, P.R. China E-mail: pro.haiyong@yahoo.com

Key words: sagittal alignment, spinopelvic, adult spine, Chinese populations

\section{Introduction}

The primary goal of treating adult spinal diseases is to reconstruct the overall balance of the spine, relieve pain symptoms and improve patients' quality of life. The effects of spinopelvic sagittal balance on the prognosis and treatment of adult spinal diseases have been investigated in previous reports (1-3). In the adult body, spinal sagittal balance is not determined solely by spinal sagittal shape; the pelvis is also able to adjust the overall body balance and serves a key role in spinal sagittal balance (1). Clinical studies have demonstrated that there are different characteristic changes to spinopelvic sagittal balance parameters in patients with spine-associated degenerative diseases (1-3). However, little is known about spine-pelvic sagittal balance in asymptomatic adults.

Sagittal plane contour serves a vital role in maintaining a balanced posture with minimum energy expenditure and pain (1-5). A number of important spinopelvic parameters, including pelvic incidence (PI), sacral slope (SS), lumbar lordosis (LL), thoracic kyphosis (TK) and cervical lordosis (CL) are used to evaluate spine and pelvis function (6). A database containing these parameters may be used to estimate the diseased spine and pelvis and may serve as reference for patients with similar spinal diseases (5). However, bias of these parameters for asymptomatic patients exist, therefore, further study with a large sample size is required. Furthermore, studies are often limited by small sample sizes.

A number of studies have been performed assessing the morphology and orientation of the spine and pelvis in asymptomatic adults. These studies covered a wide range of populations, including European Caucasian (7), Japanese (8), Korean (9) and Chinese (10). Together, the results of these studies have suggested that these parameters (PI, SS, LL, TK and CL) are correlated with age, sex and ethnicity, even though normal values fluctuated.

The aim of the present study was to evaluate spinopelvic morphology in asymptomatic Chinese Han patients and to identify correlations between these parameters (PI, SS, LL, TK and CL) and their association with patient characteristics.

\section{Materials and methods}

Subjects. A total of 85 asymptomatic Chinese Han volunteers (39 males, 46 females) were enrolled in the present study 
Table I. Measurement methods for spinopelvic parameters.

Spinopelvic parameter

Pelvic incidence

Pelvic tilt

Sacral slope

Lumbar lordosis

Thoracic kyphosis

Cervical lordosis

Sagittal vertical axis

Lumbopelvic lordosis angle

Spino-sacral angle

Sacral pelvic angle
Measurement method

Angle between the line perpendicular to the sacral plate at its midpoint and the line connecting the point to the middle axis of the femoral heads.

Angle between the line connecting the midpoint of the sacral plate to the axis of the femoral heads and the vertical axis.

Angle between the superior endplate of $\mathrm{S} 1$ and a horizontal axis.

Angle from the upper endplate of L1 to the upper end plate of S1.

Angle between the cranial endplate of T4 and the caudal endplate of T12.

Angle between the horizontal plane and the line joining the centre of $\mathrm{C} 7$ and the centre of the sacral endplate.

Horizontal distance between the $\mathrm{C} 7$ plumb line and the posterior superior corner of the superior margin of S1.

Angle between the PR line and a tangent line along the inferior endplate of T12 vertebral body. Angle subtended by the upper sacral endplate and the line from the centre of C7 vertebral body to the centre of the upper sacral endplate.

Angle between PR line and the line perpendicular to the sacral endplate.

Table II. Basic participant characteristics and measured spinopelvic parameters.

\begin{tabular}{|c|c|c|c|c|c|}
\hline Parameters & Mean & Range & Standard deviation & Standard error & $95 \%$ Confidence interval \\
\hline Age (years) & 46.0 & $21-65$ & 14.1 & 1.53 & $43.0-49.0$ \\
\hline Body mass index $\left(\mathrm{kg} / \mathrm{m}^{2}\right)$ & 21.7 & $17.3-24.5$ & 1.7 & 0.19 & $21.3-22.1$ \\
\hline Pelvic incidence $\left({ }^{\circ}\right)$ & 51.1 & $35-70$ & 8.2 & 0.89 & $49.4-52.9$ \\
\hline Pelvic tilt $\left({ }^{\circ}\right)$ & 18.5 & $3-50$ & 8.0 & 0.86 & $16.7-20.2$ \\
\hline Sacral slope $\left({ }^{\circ}\right)$ & 32.8 & $20-53$ & 6.3 & 0.69 & $31.4-34.2$ \\
\hline Lumbar lordosis $\left({ }^{\circ}\right)$ & 36.7 & $10-58$ & 11.8 & 1.28 & $34.1-39.2$ \\
\hline Thoracic kyphosis $\left({ }^{\circ}\right)$ & 20.8 & $1-57$ & 11.3 & 1.23 & $18.3-23.2$ \\
\hline Cervical lordosis $\left({ }^{\circ}\right)$ & 11.2 & $0-50$ & 9.7 & 1.06 & $9.1-13.3$ \\
\hline Sagittal vertical axis (mm) & 14.9 & $-87.0-116.1$ & 39.6 & 4.35 & $4.9-22.2$ \\
\hline Lumbopelvic lordosis angle $\left({ }^{\circ}\right)$ & 75.6 & $50-90$ & 9.8 & 1.07 & $73.5-77.7$ \\
\hline Spino-sacral angle $\left({ }^{\circ}\right)$ & 122.9 & $105-143$ & 7.8 & 0.84 & $121.2-124.6$ \\
\hline Sacral pelvic angle $\left({ }^{\circ}\right)$ & 54.3 & $40-74$ & 7.1 & 0.77 & $52.8-55.8$ \\
\hline
\end{tabular}

between August 2015 and August 2017 from the Beijing Tiantan Hospital (Beijing, China). The present study was approved by the Ethical Research Committee of Beijing Tiantan Hospital (Beijing, China). All participants provided informed consent. Inclusion criteria were as follows: 21-65 years of age, no prior spinal surgery, no history of lower back pain (except for occasional episodes) for $\geq 6$ months prior to participation and no history of hip, pelvic or lower limb disorders. The mean age, weight and height of participants were $46.0 \pm 14.1$ years, $61.2 \pm 8.3 \mathrm{~kg}, 167.7 \pm 8.3 \mathrm{~cm}$, respectively. The body mass index (BMI) of each participant was also calculated and recorded.

Radiography. Whole-body radiographs prior to surgery were taken of each participant, including frontal and left lateral images to cover the full spine and the hip joints (11). Hips and knees were fully extended in both frontal and left lateral direction (12). As described previously $(8-10,13), 10$ representative radiographic parameters were assessed: PI, pelvic tilt (PT), SS, LL, TK, CL, sagittal vertical axis (SVA), lumbopelvic lordosis angle (PR-T12), spino-sacral angle (SSA) and sacral pelvic angle (SPA; Fig. 1; Table I). Radiography was performed using the pelvic radius measurement technique (14). Radiographs were stored in a picture archiving communications system (PACS; Concord Technology (Tianjian) Co., Ltd. (Tianjin, China) in Digital Imaging and Communications in Medicine (DICOM) format. Parameters associated with sagittal alignment were measured using the ruler and protractor function in Neusoft PACS (Concord Technology (Tianjian) Co., Ltd.). Radiographs were analyzed by two orthopedic doctors. Using PACS software (Version 6.0; Concord Technology (Tianjian) Co., Ltd.), the inter- and intraobserver reliability were evaluated using inter- and 

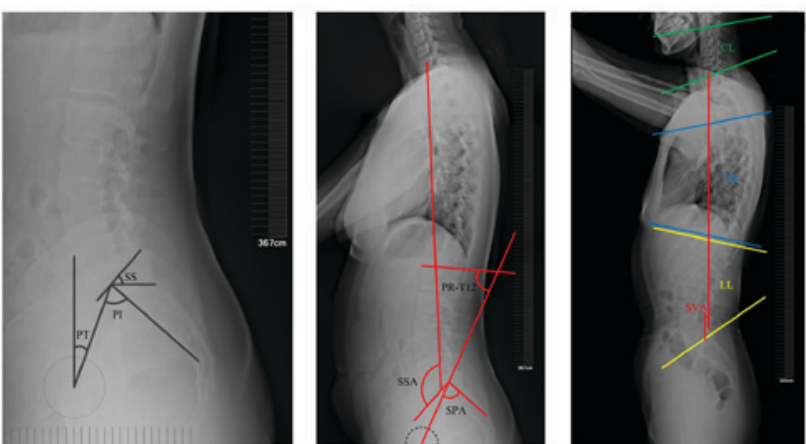

Figure 1. Measurement methods for spinopelvic parameters. PI, pelvic incidence; PT, pelvic tilt; SS, sacral slope; LL, lumbar lordosis; CL, cervical lordosis; TK, thoracic kyphosis; SVA, sagittal vertical axis; PR-T12, lumbopelvic lordosis angle; SSA, spino-sacral angle; SPA, sacral pelvic angle.

intraclass correlation coefficients and radiological parameter reliabilities were $>0.900$.

Statistical analysis. Data were analyzed using SPSS version 13.0 (SPSS Inc., Chicago, IL, USA). All quantitative data were expressed as the mean \pm standard deviation. An unpaired Student's t-test was performed to compare male and female results. Correlations were evaluated using Pearson's correlation coefficient. The inter- and intraobserver/class correlation coefficients were assessed for angles, levels and distances of parameters. $\mathrm{P}<0.05$ was considered to indicate a statistically significant difference.

\section{Results}

Clinical data. A total of 39 males and 46 females were enrolled in the present study, with mean ages of $50.9 \pm 13.2$ and $41.8 \pm 13.6$ years, respectively. Mean values and $95 \%$ confidence intervals of PI, PT, SS, LL, TK, CL, SVA, PR-T12, SSA and SPA, as well as patient characteristics, are presented in Table II.

Ethnic variation between spinopelvic characteristics. The spinopelvic characteristics of patients enrolled in the present study are presented in Table III along with those of other reported populations, including Korean, Japanese, Caucasian and another Chinese Han population (7-10).

Sex differences in spinopelvic parameters. Sex differences in spinopelvic parameters are presented in Table IV. Female participants were observed to have decreased PT, TK and CL values compared with males. In addition, females demonstrated higher values of PI, SS, LL, PR-T12 and SPA, but these differences were not statistically significant. However, SVA and SSA in females were significantly higher than those in males $(\mathrm{P}<0.05)$.

BMI affects specific spinopelvic parameters. Based on previous studies, a BMI of 22.0 was chosen as a boundary $(9,10)$. A comparison between patients with BMI $>22.0$ and those with $<22.0$ is presented in Table $\mathrm{V}$. The results revealed that LL and TK markedly differed between groups. The LL value of patients with a BMI of $>22.0$ were

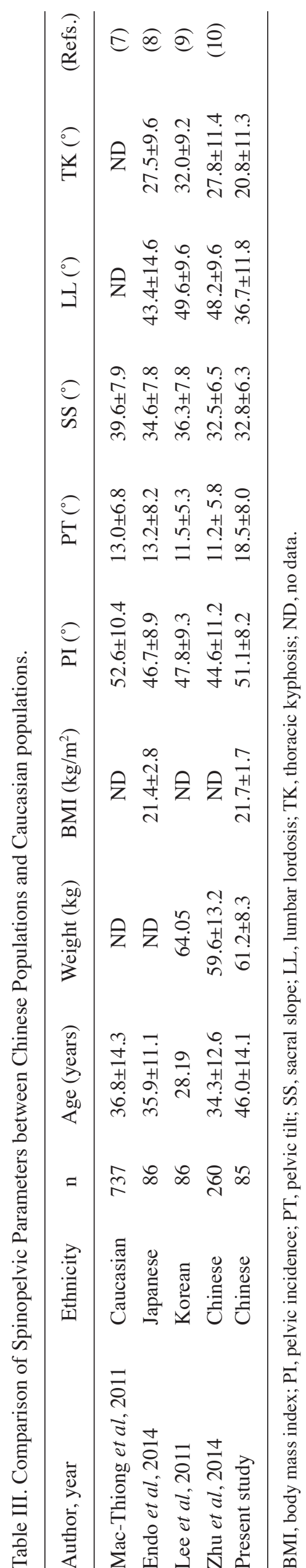


Table IV. Sex distribution of spinopelvic parameters.

\begin{tabular}{lccr}
\hline Parameters & Male $(\mathrm{n}=39)$ & Female $(\mathrm{n}=46)$ & P-value \\
\hline Age $($ years $)$ & $50.9 \pm 13.2$ & $41.8 \pm 13.6$ & 0.0026 \\
Height $(\mathrm{cm})$ & $174 \pm 4$ & $162 \pm 4$ & 0.0000 \\
Weight $(\mathrm{kg})$ & $68.7 \pm 4.1$ & $54.9 \pm 5.0$ & 0.0000 \\
Body mass index $\left(\mathrm{kg} / \mathrm{m}^{2}\right)$ & $22.6 \pm 1.1$ & $20.9 \pm 1.8$ & 0.0000 \\
Pelvic incidence $\left({ }^{\circ}\right)$ & $50.7 \pm 8.9$ & $51.5 \pm 7.7$ & 0.6531 \\
Pelvic tilt $\left({ }^{\circ}\right)$ & $19.2 \pm 9.0$ & $17.8 \pm 7$ & 0.4216 \\
Sacral slope $\left({ }^{\circ}\right)$ & $31.5 \pm 5.8$ & $33.9 \pm 6.7$ & 0.0822 \\
Lumbar lordosis $\left({ }^{\circ}\right)$ & $35.1 \pm 11.8$ & $38.0 \pm 11.8$ & 0.2507 \\
Thoracic kyphosis $\left({ }^{\circ}\right)$ & $22.9 \pm 13.4$ & $19 \pm 8.9$ & 0.1158 \\
Cervical lordosis $\left({ }^{\circ}\right)$ & $12.1 \pm 10.2$ & $10.5 \pm 9.4$ & 0.4539 \\
Sagittal vertical axis $(\mathrm{mm})$ & $25.6 \pm 42.5$ & $35.2 \pm 3.4$ & 0.0099 \\
Lumbopelvic lordosis angle $\left({ }^{\circ}\right)$ & $73.9 \pm 11.4$ & $77.1 \pm 8.2$ & 0.1429 \\
Spino-sacral angle $\left(^{\circ}\right)$ & $120.8 \pm 7.0$ & $124.7 \pm 8.0$ & 0.0218 \\
Sacral-pelvic angle $\left({ }^{\circ}\right)$ & $54.4 \pm 7$ & $54.3 \pm 7.2$ & 0.9364 \\
\hline
\end{tabular}

Table V. Comparison of spinopelvic parameters by BMI.

\begin{tabular}{lcc}
\hline Parameters & BMI $(\leq 22.0)$ & BMI $(>22.0)$ \\
\hline BMI $\left(\mathrm{kg} / \mathrm{m}^{2}\right)$ & $20.1 \pm 1.2$ & $23.0 \pm 0.7$ \\
Pelvic incidence $\left({ }^{\circ}\right)$ & $52.0 \pm 7.8$ & $50.4 \pm 8.5$ \\
Pelvic tilt $\left(^{\circ}\right)$ & $18.5 \pm 7.1$ & $18.4 \pm 8.7$ \\
Sacral slope $\left({ }^{\circ}\right)$ & $33.4 \pm 6.9$ & $32.3 \pm 5.8$ \\
Lumbar lordosis $\left({ }^{\circ}\right)$ & $38.8 \pm 11.3$ & $34.9 \pm 12.1^{\mathrm{a}}$ \\
Thoracic kyphosis $\left(^{\circ}\right)$ & $19.4 \pm 11.0$ & $22.0 \pm 11.5^{\mathrm{a}}$ \\
Cervical lordosis $\left({ }^{\circ}\right)$ & $10.9 \pm 10.5$ & $11.5 \pm 9.1$
\end{tabular}

BMI, body mass index; ${ }^{\mathrm{a}} \mathrm{P}<0.05$ vs. BMI $(\leq 22.0)$.

significantly lower than those with a score of $<22.0(\mathrm{P}<0.05)$. Furthermore, patients with a BMI of $>22.0$ exhibited a significantly higher TK value $(\mathrm{P}<0.05)$ compared with those with a BMI of $<22.0$. However, there no significant differences were identified regarding PI, PT, SS or CL between the two groups.

Correlation between spinopelvic parameters. The correlation matrix for spinopelvic parameters is presented in Table VI. PI was correlated with PT, SS, PR-T12 and SPA.PT was correlated with PI, SS, LL, PR-T12, SSA and SPA. SS was correlated with PI, PT, LL, PR-T12 and SSA. LL was correlated with PT, SS, PR-T12 and SSA. TK and CL were correlated with each other. SVA was correlated with PR-T12. PR-T12 was correlated with PI, PT, SS, LL, SVA, SSA and SPA. SSA was correlated with PT, SS, LL and PR-T12. SPA was correlated with PI, PT and PR-T12.

Age differences in spinopelvic parameters. Correlations between age and spinopelvic parameters are presented in Table VII. Age was positively correlated with TK, CL and SVA, while it was negatively correlated with LL, PR-T12 and
SSA. No significant correlation was observed for age with PI, PT, SS or SPA.

\section{Discussion}

A number of studies have reported that PI is an important parameter for determining the shape of sagittal curvature $(6,15-17)$. Studying the corresponding spinopelvic parameters in asymptomatic adults may allow for the development of suitable spinal surgical protocols. These parameters (PI, SS, LL, TK and CL) may aid the understanding of degenerative spine disorders and scoliosis (1-3). LL can be predicted based on preoperative PI and TK (18). Unsuccessful TK restoration may be associated with large preoperative PI and PT (19). A number of studies have focused on normal spinal alignment and evaluated the correlation between spinopelvic parameters $(7-10,20)$. However, a small sample size has often led to the bias of these parameters. Consequently, more data are required to improve prognostic tools.

A total of 85 asymptomatic Chinese Han adults were enrolled in the present study. A series of spinopelvic parameters were measured and it was revealed that the mean value of PI, one of the important parameters that is not affected by gesture or spinal pathology (6), was increased compared with previous reports on Chinese, Korean and Japanese populations (8-10). The PI recorded in the present study was similar to that reported in a paper involving Caucasian participants (7). The mean value for PT was much higher compared with the results of a Caucasian population study and three other Asian population studies (7-10). LL and TK exhibited the opposite tendency; these spinopelvic parameters were decreased in the current study compared with a Caucasian and three Asian populations. SS was comparable with the previous results of these studies (7-10).

One of the major contributing factors affecting the parameters was participant age. Compared with the mean age of

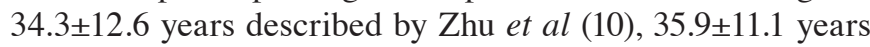


Table VI. Correlation matrix for the spinopelvic parameters.

\begin{tabular}{|c|c|c|c|c|c|c|c|c|c|c|}
\hline Parameters & PI & PT & SS & LL & TK & $\mathrm{CL}$ & SVA & PR-T12 & SSA & SPA \\
\hline PI & 1 & & & & & & & & & \\
\hline PT & $0.690^{\mathrm{b}}$ & 1 & & & & & & & & \\
\hline SS & $0.426^{\mathrm{b}}$ & $-0.349^{b}$ & 1 & & & & & & & \\
\hline LL & 0.178 & $-0.244^{a}$ & $0.534^{b}$ & 1 & & & & & & \\
\hline TK & -0.019 & -0.009 & 0 & 0.078 & 1 & & & & & \\
\hline CL & -0.002 & 0.042 & -0.039 & -0.025 & $0.272^{\mathrm{a}}$ & 1 & & & & \\
\hline SVA & 0.094 & 0.014 & 0.112 & -0.161 & 0.211 & 0.023 & 1 & & & \\
\hline PR-T12 & $-0.322^{\mathrm{b}}$ & $-0.641^{\mathrm{b}}$ & $0.394^{b}$ & $0.510^{\mathrm{b}}$ & 0.047 & -0.007 & $-0.273^{\mathrm{a}}$ & 1 & & \\
\hline SSA & 0.053 & $-0.277^{\mathrm{a}}$ & $0.406^{\mathrm{b}}$ & $0.486^{\mathrm{b}}$ & -0.053 & -0.157 & -0.209 & $0.311^{\mathrm{b}}$ & 1 & \\
\hline SPA & $0.675^{\mathrm{b}}$ & $0.544^{\mathrm{b}}$ & 0.199 & 0.203 & 0.064 & -0.004 & 0.089 & $-0.278^{a}$ & 0.016 & 1 \\
\hline
\end{tabular}

${ }^{a} \mathrm{P}<0.05$ and ${ }^{\mathrm{b}} \mathrm{P}<0.01$. PI, pelvic incidence; PT, pelvic tilt; SS, sacral slope; LL, lumbar lordosis; CL, cervical lordosis; TK, thoracic kyphosis; SVA, sagittal vertical axis; PR-T12, lumbopelvic lordosis angle; SSA, spino-sacral angle; SPA, sacral pelvic angle.

Table VII. Pearson correlation between spinopelvic parameters and age.

\begin{tabular}{lcc}
\hline Parameters & Coefficient & P-value \\
\hline Pelvic incidence $\left({ }^{\circ}\right)$ & 0.105 & 0.337 \\
Pelvic tilt $\left(^{\circ}\right)$ & 0.116 & 0.290 \\
Sacral slope $\left({ }^{\circ}\right)$ & 0.018 & 0.868 \\
Lumbar lordosis $\left({ }^{\circ}\right)$ & -0.251 & 0.020 \\
Thoracic kyphosis $\left({ }^{\circ}\right)$ & 0.225 & 0.039 \\
Cervical lordosis $\left({ }^{\circ}\right)$ & 0.180 & 0.098 \\
Sagittal vertical axis (mm) & 0.459 & 0.000 \\
Lumbopelvic lordosis angle $\left(^{\circ}\right)$ & -0.296 & 0.006 \\
Spino-sacral angle $\left(^{\circ}\right)$ & -0.222 & 0.041 \\
Sacral pelvic angle $\left({ }^{\circ}\right)$ & -0.016 & 0.888 \\
\hline
\end{tabular}

reported by Endo et al (8) and 28.2 reported by Lee et al (9), participants in the current study had a mean age of $46.0 \pm 14.1$ years. Several recent studies have demonstrated that sagittal spinopelvic alignment varies with age and that these parameters (PI, SS, LL, TK and CL) gradually deteriorate with age (21-23). A weak correlation was observed between age and spinopelvic parameters by Mac-Thiong et al (7), while Zhu et al (10) reported that PT significantly increased with age. Hamerberg and Wood (24) demonstrated that there was no association between age and spinopelvic parameters. The current study suggested that age was positively correlated with TK, CL and SVA, while negatively correlated with LL, PR-T12 and SSA. SS was suggested to be independent of age. These results suggest that age should be taken into consideration prior to establishing a surgical plan for patients with spinal deformities.

Ethnicity was reported to be associated with sagittal parameters of the spine in several studies $(9,10,25-27)$. When comparing the results of the current study with previous reports of Asian and Caucasian populations, SS was found to have an association with ethnicity. The mean value of SS in the current study was $32.8 \pm 6.3^{\circ}$, which is similar to that reported for a Japanese $\left(34.6 \pm 7.8^{\circ}\right)(8)$, Korean $\left(36.3 \pm 7.8^{\circ}\right)(9)$ and other Chinese $\left(32.5 \pm 6.5^{\circ}\right)$ (10) populations, but decreased compared with the SS reported for a Caucasian population $\left(39.6 \pm 7.9^{\circ}\right)(7)$. These findings were consistent with the data reported by Zhu's et al (10). For patients with spinal deformities, ethnicity may be considered an important factor for the selection of the appropriate treatment strategy.

The sex distribution of spinopelvic parameters was evaluated. SS and PI were significantly influenced by sex as suggested by Vialle et al (12) and Vira et al (28), who reported that men exhibit increased knee flexion and women exhibit increased pelvic tilt and hip extension. Janssen et al (29) and Zhu et al (10) reported no significant differences in SS, PT and PI between males and females. In the present study, it was determined that SSA and SVA were significantly different in males compared with females.

The effect of BMI on spinopelvic parameters was further evaluated. It was suggested that LL and TK may be associated with BMI, while other parameters exhibited no association. Individual physiological characters, including sex and BMI, may be considered as effectors of spinopelvic parameters.

Associations between spinopelvic parameters were evaluated. PI was considered to be the most important factor among all parameters. It was concluded that PI was associated with SSA, PR-T12, PT and SPA. This suggests that PI measurement may be substituted with SSA, PR-T12 or SPA measurements, as these parameters are more convenient to measure and may make the evaluation more objective.

To the best of the authors' knowledge, there have been several studies on the sagittal alignment of the spine and pelvis in asymptomatic adults, conducted in various countries and suggesting various correlations between parameters $(7-10,20)$. In the present study, it was suggested that age was positively correlated with TK, CL and SVA, while it was negatively correlated with LL, PR-T12 and SSA. SSA and SVA were significantly different in males compared with females, while other spinopelvic parameters exhibited no significant differences. LL and TK were suggested to potentially be associated with BMI, while other parameters did not appear 
to be correlated with BMI. The results of the present study are comparable with those of previous studies and different in others. However, the results may offer significant qualitative and quantitative references for treating patients with spinal degenerative diseases.

The present study was not without limitations. For instance, the study size was too small and so was not representative of the general population. Furthermore, although participants were screened in compliance with the outlined rules, data obtained from certain individuals did not fall within the expected ranges. Future studies should recruit a wider range of participants to improve the results.

In summary, the present study suggests that certain spinopelvic parameters are correlated with age, while others may be associated with ethnicity, sex and BMI. These results may provide a surgical reference for restoring LL or similar diseases.

\section{Acknowledgements}

Not applicable.

\section{Funding}

The present study was supported by the Beijing Municipal Administration of Hospitals Incubating Program (grant no. PX2017066).

\section{Availability of data and materials}

All data generated or analyzed during the present study are included in this published article.

\section{Authors' contributions}

$\mathrm{ZZ}$ and YH designed and planned the study. YB collected the data. BW analyzed the data and performed statistical analyses. ML and YL helped with data interpretation. ZZ prepared the manuscript and YL performed a literature analysis. All authors read and approved the final manuscript.

\section{Ethics approval and consent to participate}

The present study was approved by the Ethics Committee of Beijing Chaoyang Hospital (Beijing, China). Written informed consent was obtained from the participants.

\section{Patient consent for publication}

Not applicable.

\section{Competing interests}

The authors declare that they have no competing interests.

\section{References}

1. Roussouly P and Pinheiro-Franco JL: Sagittal parameters of the spine: Biomechanical approach. Eur Spine J 5: 578-585, 2011

2. Vaz G, Roussouly P, Berthonnaud E and Dimnet J: Sagittal morphology and equilibrium of pelvis and spine. Eur Spine J 11: 80-87, 2002.
3. Cil A, Yazici M, Uzumcugil A, Kandemir U, Alanay A, Alanay Y, Acaroglu RE and Surat A: The evolution of sagittal segmental alignment of the spine during childhood. Spine (Phila Pa 1976) 30: 93-100, 2005.

4. Jackson RP, Kanemura T, Kawakami N and Hales C: Lumbopelvic lordosis and pelvic balance on repeated standing lateral radiographs of adult volunteers and untreated patients with constant low back pain. Spine (Phila Pa 1976) 25: 575-586, 2000.

5. Jackson RP and Hales C: Congruent spinopelvic alignment on standing lateral radiographs of adult volunteers. Spine (Phila Pa 1976) 25: 2808-2815, 2000

6. Legaye J, Duval-Beaupere G, Hecquet J and Marty C: Pelvic incidence: A fundamental pelvic parameter for three-dimensional regulation of spinal sagittal curves. Eur Spine J 7: 99-103, 1998.

7. Mac-Thiong JM, Roussouly P, Berthonnaud E and Guigui P: Ageand sex-related variations in sagittal sacropelvic morphology and balance in asymptomatic adults. Eur Spine J 20 (Suppl 5): S572-S577, 2011.

8. Endo K, Suzuki H, Nishimura H, Tanaka H, Shishido T and Yamamoto K: Characteristics of sagittal spino-pelvic alignment in Japanese young adults. Asian Spine J 8: 599-604, 2014.

9. Lee CS, Chung SS, Kang KC, Park SJ and Shin SK: Normal patterns of sagittal alignment of the spine in young adults radiological analysis in a Korean population. Spine (Phila Pa 1976) 36: E1648-E1654, 2011.

10. Zhu Z, Xu L, Zhu F, Jiang L, Wang Z, Liu Z, Qian BP and Qiu Y: Sagittal alignment of spine and pelvis in asymptomatic adults: Norms in Chinese populations. Spine (Phila Pa 1976) 39: E1-E6, 2014.

11. Park SA, Kwak DS, Cho HJ and Min DU: Changes of spinopelvic parameters in different positions. Arch Orthop Trauma Surg 137: 1223-1232, 2017

12. Vialle R, Levassor N, Rillardon L, Templier A, Skalli W and Guigui P: Radiographic analysis of the sagittal alignment and balance of the spine in asymptomatic subjects. J Bone Joint Surg Am 87: 260-267, 2005.

13. Suzuki H, Endo K, Mizuochi J, Kobayashi H, Tanaka H and Yamamoto K: Clasped position for measurement of sagittal spinal alignment. Eur Spine J 19: 782-786, 2010.

14. Jackson RP, Peterson MD, McManus AC and Hales C: Compensatory spinopelvic balance over the hip axis and better reliability in measuring lordosis to the pelvic radius on standing lateral radiographs of adult volunteers and patients. Spine (Phila Pa 1976) 23: 1750-1767, 1998

15. Boulay C, Tardieu C, Hecquet J, Benaim C, Mouilleseaux B, Marty C, Prat-Pradal D, Legaye J, Duval-Beaupere G and Pelissier J: Sagittal alignment of spine and pelvis regulated by pelvic incidence: Standard values and prediction of lordosis. Eur Spine J 15: 415-422, 2006.

16. Gelb DE, Lenke LG, Bridwell KH, Blanke K and McEnery KW: An analysis of sagittal spinal alignment in 100 asymptomatic middle and older aged volunteers. Spine (Phila Pa 1976) 20: 1351-1358, 1995.

17. Berthonnaud E, Dimnet J, Roussouly P and Labelle H: Analysis of the sagittal balance of the spine and pelvis using shape and orientation parameters. J Spinal Disord Tech 18: 40-47, 2005.

18. Rose PS, Bridwell KH, Lenke LG, Cronen GA, Mulconrey DS, Buchowski JM and Kim YJ: Role of pelvic incidence, thoracic kyphosis, and patient factors on sagittal plane correction following pedicle subtraction osteotomy. Spine (Phila $\mathrm{Pa}$ 1976) 34: 785-791, 2009.

19. Lafage V, Ames C, Schwab F, Klineberg E, Akbarnia B, Smith J, Boachie-Adjei O, Burton D, Hart R, Hostin R, et al: Changes in thoracic kyphosis negatively impact sagittal alignment after lumbar pedicle subtraction osteotomy: A comprehensive radiographic analysis. Spine (Phila Pa 1976) 37: E180-E187, 2012.

20. Chanplakorn P, Wongsak S, Woratanarat P, Wajanavisit W and Laohacharoensombat W: Lumbopelvic alignment on standing lateral radiograph of adult volunteers and the classification in the sagittal alignment of lumbar spine. Eur Spine J 20: 706-712, 2011.

21. Lafage R, Schwab F, Challier V, Henry JK, Gum J, Smith J, Hostin R, Shaffrey C, Kim HJ, Ames C, et al: Defining Spino-Pelvic alignment thresholds: Should operative goals in adult spinal deformity surgery account for age? Spine (Phila Pa 1976) 41: 62-68, 2016.

22. Hasegawa K, Okamoto M, Hatsushikano S, Shimoda H, Ono M and Watanabe K: Normative values of spino-pelvic sagittal alignment, balance, age, and health-related quality of life in a cohort of healthy adult subjects. Eur Spine J 25: 3675-3686, 2016. 
23. Banno T, Togawa D, Arima $\mathrm{H}$, Hasegawa T, Yamato $\mathrm{Y}$, Kobayashi S, Yasuda T, Oe S, Hoshino H and Matsuyama Y: The cohort study for the determination of reference values for spinopelvic parameters (T1 pelvic angle and global tilt) in elderly volunteers. Eur Spine J 25: 3687-3693, 2016.

24. Hammerberg EM and Wood KB: Sagittal profile of the elderly. J Spinal Disord Tech 16: 44-50, 2003.

25. Gilsanz V, Skaggs DL, Kovanlikaya A, Sayre J, Loro ML, Kaufman F and Korenman SG: Differential effect of race on the axial and appendicular skeletons of children. J Clin Endocrinol Metab 83: 1420-1427, 1998

26. Seeman E: Growth in bone mass and size-are racial and gender differences in bone mineral density more apparent than real? J Clin Endocrinol Metab 83: 1414-1419, 1998

27. Lonner BS, Auerbach JD, Sponseller P, Rajadhyaksha AD and Newton PO: Variations in pelvic and other sagittal spinal parameters as a function of race in adolescent idiopathic scoliosis. Spine (Phila Pa 1976) 35: E374-E377, 2010.
28. Vira S, Diebo BG, Spiegel MA, Liabaud B, Henry JK, Oren JH, Lafage R, Tanzi EM, Protopsaltis TS, Errico TJ, et al: Is there a Gender-Specific full body sagittal profile for different spinopelvic relationships? A study on Propensity-Matched cohorts. Spine Deform 4: 104-111, 2016.

29. Janssen MM, Drevelle X, Humbert L, Skalli W and Castelein RM: Differences in male and female spino-pelvic alignment in asymptomatic young adults: A three-dimensional analysis using upright low-dose digital biplanar X-rays. Spine (Phila Pa 1976) 34: E826-E832, 2009.

This work is licensed under a Creative Commons Attribution-NonCommercial-NoDerivatives 4.0 International (CC BY-NC-ND 4.0) License. 